\title{
Novel dynamic outcome indicators and clinical endpoints in myelodysplastic syndrome; the European LeukemiaNet MDS Registry and MDS-RIGHT project perspective
}

Haematologica 2020

Volume 105(11):2516-2523

\section{Correspondence:}

THEO DE WITTE,

theo.dewitte@radboudumc.nl

Received: July 15, 2020.

Accepted: August 17, 2020.

Pre-published: September 21, 2020.

doi:10.3324/haematol.2020.266817

(C)2020 Ferrata Storti Foundation

Material published in Haematologica is covered by copyright. All rights are reserved to the Ferrata Storti Foundation. Use of published material is allowed under the following terms and conditions:

https://creativecommons.org/licenses/by-nc/4.0/legalcode. Copies of published material are allowed for personal or internal use. Sharing published material for non-commercial purposes is subject to the following conditions:

https://creativecommons.org/licenses/by-nc/4.0/legalcode, sect. 3. Reproducing and sharing published material for commercial purposes is not allowed without permission in writing from the publisher.
Theo de Witte, ${ }^{1 *}$ Luca Malcovati, ${ }^{2 \star}$ Pierre Fenaux, ${ }^{3}$ David Bowen, ${ }^{4}$ Argiris Symeonidis, ${ }^{5}$ Moshe Mittelman, ${ }^{6}$ Reinhard Stauder, ${ }^{7}$ Guillermo Sanz, ${ }^{8}$ Jaroslav Čermák, ${ }^{9}$ Saskia Langemeijer, ${ }^{10}$ Eva Hellström-Lindberg, ${ }^{11}$ Ulrich Germing, ${ }^{12}$ Mette Skov Holm, ${ }^{13}$ Krzysztof Mądry, ${ }^{14}$ Aurelia Tatic, ${ }^{15}$ António Medina Almeida, ${ }^{16}$ Aleksandar Savic, ${ }^{17}$ Inga Mandac Rogulj, ${ }^{18}$ Raphael Itzykson, ${ }^{3}$ Marlijn Hoeks, ${ }^{10}$ Hege Gravdahl Garelius, ${ }^{19}$ Dominic Culligan, ${ }^{20}$ Ioannis Kotsianidis, ${ }^{21}$ Lionel Ades, ${ }^{3}$ Arjan A. van de Loosdrecht, ${ }^{22}$ Corine van Marrewijk, ${ }^{10} \mathrm{Ge} \mathrm{Yu},{ }^{23}$ Simon Crouch, ${ }^{23}$ Alex Smith ${ }^{23}$; on behalf of the EUMDS Registry Participants

${ }^{1}$ Department of Tumor Immunology - Nijmegen Center for Molecular Life Sciences, Radboud University Medical Center, Nijmegen, the Netherlands; ${ }^{2}$ Department of Hematology Oncology, Fondazione IRCCS Policlinico San Matteo, University of Pavia, Pavia, Italy; '3Service d'Hématologie, Hôpital Saint-Louis, Assistance Publique des Hôpitaux de Paris (AP-HP) and Université Paris 7, Paris, France; ${ }^{4}$ St. James's Institute of Oncology, Leeds Teaching Hospitals, Leeds, UK; ${ }^{5}$ Department of Medicine, Division of Hematology, University of Patras Medical School, Patras, Greece; ${ }^{6}$ Department of Medicine A, Tel Aviv Sourasky (Ichilov) Medical Center and Sackler Medical Faculty, Tel Aviv University, Tel Aviv, Israel; ' Department of Internal Medicine V (Haematology and Oncology), Innsbruck Medical University, Innsbruck, Austria; ${ }^{8}$ Department of Haematology, Hospital Universitario y Politécnico La Fe, Valencia, and CIBERONC, Madrid, Spain; ${ }^{9}$ Department of Clinical Hematology, Inst. of Hematology and Blood Transfusion, Praha, Czech Republic; ${ }^{10}$ Department of Hematology, Radboud University Medical Center, Nijmegen, the Netherlands; ${ }^{11}$ Department of Medicine, Division Hematology, Karolinska Institutet, Stockholm, Sweden; ${ }^{12}$ Department of Haematology, Oncology and Clinical Immunology, Universitätsklinik Düsseldorf, Düsseldorf, Germany; ${ }^{13}$ Department of Haematology, Aarhus University Hospital, Aarhus, Denmark; ${ }^{14}$ Department of Haematology, Oncology and Internal Medicine, Warszawa Medical University, Warszawa, Poland; ${ }^{15}$ Center of Hematology and Bone Marrow Transplantation, Fundeni Clinical Institute, Bucharest, Romania; ${ }^{16}$ Department of Clinical Hematology, Hospital da Luz, Lisbon, Portugal; ${ }^{17}$ Clinic of Hematology - Clinical Center of Vojvodina, Faculty of Medicine, University of Novi Sad, Novi Sad, Serbia; ${ }^{18}$ Department of Internal Medicine, Division of Hematology, Merkur University Hospital, Zagreb, Croatia; ${ }^{19}$ Department of Specialist Medicine, Sahlgrenska University Hospital, Göteborg, Sweden; ${ }^{20}$ Department of Haematology, Aberdeen Royal Infirmary, Aberdeen, UK; ${ }^{21}$ Department of Hematology, Democritus University of Thrace Medical School, University Hospital of Alexandroupolis, Alexandroupolis, Greece; ${ }^{22}$ Department of Hematology - Cancer Center Amsterdam, Amsterdam UMC, Location VU University Medical Center, Amsterdam, the Netherlands and ${ }^{23}$ Epidemiology and Cancer Statistics Group, Department of Health Sciences, University of York, York, UK

*TdW and LM both contributed equally as co-first authors.

\section{Introduction}

Myelodysplastic syndromes (MDS) are chronic bone marrow (BM) disorders characterized by peripheral blood cytopenias, predominantly in older persons with an average age at diagnosis of 75 years. ${ }^{1,2}$ The natural history of MDS is heterogeneous, ranging from indolent conditions to forms similar to acute myeloid leukemia (AML). In 1997, an International Prognostic Scoring System (IPSS) was established based on the percentage of BM blasts, number of cytopenias and cytogenetic characteristics. ${ }^{3}$ In 2012, this prognostic scoring system was refined (IPSS-R) to include better categorization of cytopenias, blast cell percentage and an improved risk stratification of the cytogenetic risk groups. ${ }^{4}$ Generally, MDS are divided into two prognostic groups: lower-risk MDS (LR-MDS) with patients from the (very) low risk or intermediate risk groups, and higher-risk MDS (HR-MDS) with patients from the (very) poor risk groups, as defined within the IPSS-R. ${ }^{2}$

The majority of patients with MDS (75\%) have LR-MDS, which was the focus of the European LeukemiaNet MDS (EUMDS) Registry until 2017. The EUMDS Registry is a pragmatic, observational study, which has collected prospectively longi- 
tudinal data from more than 2,738 patients with MDS, including 2,498 LR-MDS patients with a median age of 75 years, and a follow-up of up to 11 years, in 16 European countries plus Israel from 148 active sites (progress report as of March $1^{\text {st }}$ 2020). Progression to HR-MDS/AML has occurred in 314 LR-MDS patients (13\%), and 910 patients $(33 \%)$ had died at time of last report. Data quality control, including monitoring of both clinical performance and data collection, has been implemented since the initiation of the EUMDS Registry. New prognostic indicators in LR-MDS have been identified as part of the MDS-RIGHT project (https://mds-europe.eu/right) funded by Horizon 2020, which started in May 2015 with an overarching aim of defining and implementing more (cost)-effective and safer interventions in LR-MDS.

Symptoms of anemia, the most common cytopenia in LRMDS, accompanied by infectious or bleeding complications predominate in LR-MDS. ${ }^{5}$ About $25 \%$ of these patients develop AML, but most patients die from complications related to progressive BM failure and worsening cytopenias, and from their negative interaction with the extra-hematologic comorbidities presented by those patients of advanced age. ${ }^{6,7}$ Patients with LR-MDS are characterized by a notable reduction in health related quality of life (HRQoL).,9 Moderate to severe anemia in older individuals ( $>60$ years) leads to increased mortality both in patients with LR-MDS and in the general population. ${ }^{10,11}$ Likewise, anemia represents an unfavorable prognosticator in possible pre-MDS conditions at advanced age. ${ }^{12}$ Improving response prediction will contribute to more effective and targeted use of the available health care interventions (HCI). ${ }^{13}$

According to the available evidence- and consensusbased therapeutic guidelines, current therapeutic interventions in LR-MDS include red blood cell transfusion (RBCT), erythropoietin stimulating agents (ESA), lenalidomide, and iron chelation therapy (ICT). ${ }^{11}$ For ESA, a predictive model has identified a group of patients characterized by serum erythropoietin (EPO) levels $<500 \mathrm{mU} / \mathrm{mL}$ and a transfusion need of $<2$ units $\mathrm{RBC} /$ month with a favorable response compared to patients with higher EPO levels and/or higher numbers of RBCT/month. ${ }^{14}$ Treatment with lenalidomide is only recommended for a small subgroup of patients with partial loss of chromosome 5 (5q-) and RBCT-dependent anemia. ${ }^{15,16}$

The most frequently applied outcome parameter in this LR-MDS patient population is overall survival (OS). Analysis of the EUMDS Registry data showed that the currently available risk scoring systems, including the IPSS-R, have a better prognostic capacity for disease progression to AML as compared with OS. ${ }^{2}$ We estimated that $58 \%$ of deaths in this population are not related to disease progression, but are attributable to non-leukemic death.,17 A high proportion of patients with LR-MDS have a median survival of up to 5-10 years, meaning that clinical trials, the design of which identifies survival as a primary endpoint, may result in a potentially biased assessment of the effectiveness and evidence on the appropriate use of the available interventions. Therefore, we have explored additional relevant outcome parameters in a Delphi survey, which could be used to circumvent the limited value of survival as primary endpoint in LR-MDS. ${ }^{18}$

Additional evidence is required to extend the existing prognostic and therapeutic response indicators in the older LR-MDS population, and to identify meaningful biological and clinical endpoints, including patient-reported outcome measures and other patient-related factors. These new endpoints may provide information on the effectiveness of the available therapeutic interventions early in the natural history of the disease. These early indicators of treatment response may drive a more effective use of those interventions currently made.

Recent studies conducted on the large population of patients with LR-MDS included in the EUMDS Registry during its first 10 years of activity allowed validation of RBCT requirement and HRQoL as independent and meaningful outcome indicators and reliable measures of response to interventions, supporting their integration in the MDSCore Outcome Set (COS) in this patient population. ${ }^{18}$ In addition, prospective studies based on the unbiased dataset of the EUMDS Registry allowed identification of early response determinants for targeted use of treatment modalities, including ESA, lenalidomide, and ICT.

\section{Novel outcome indicators and meaningful early clinical endpoints in patients with lower-risk myelodysplastic syndromes}

\section{Kinetics of blood counts decrease is an independent outcome indicator in lower-risk myelodysplastic syn- dromes}

The prognosis of LR-MDS is heterogeneous. ${ }^{2}$ Early identification of patients at risk of rapid progression should rely on universal, affordable and non-invasive tools to gain acceptance in an older population often managed in community care centers. All current MDS prognostic scores rely on steady-state assessments of cytopenias, i.e., hemoglobin (Hb)-levels, neutrophil or platelet counts on the day of initial assessment. ${ }^{19}$ Time-dependent prognostic scores require repeated $\mathrm{BM}$ examinations, raising acceptability issues in this older patient population. To circumvent this limitation, we analyzed the prognostic role of the kinetics of cytopenias during the first visit interval ( 6 months) following diagnosis in LR-MDS patients prospectively included in the EUMDS registry. ${ }^{20}$ We performed a landmark analysis at the second visit, at around 6 months from diagnosis, to apply simple prognostic criteria in general clinical practice.

The results showed that a relative drop in platelets $>25 \%$ at the 6-month landmark predicted shorter 5-year OS; 22\% versus $49 \%$ in patients with platelet drop $\leq 25 \%\left(P<10^{-4}\right)$, regardless of baseline IPSS-R or absolute platelet counts. Conversely, relative neutrophil drop $>25 \%$ had no significant impact on OS. Subsequently, a classifier was built based on RBCT-dependency and relative platelet drop $>25 \%$ at landmark. Patients with none $(62 \%)$, either one of the two criteria $(27 \%)$ or both criteria $(11 \%)$ had 5 -year OS of $53.3 \%, 32.7 \%$ and $9.0 \%$, respectively $\left(P<10^{-4}\right)$ (Figure 1 ). Sensitivity analyses confirmed the applicability of this simple classifier even when follow-up visits were planned at any time during the first ten months after diagnosis, thus capturing most situations encountered in daily practice. ${ }^{20}$

Red blood cell transfusion requirement is an independent outcome indicator and freedom from transfusion a meaningful clinical endpoint in patients with lower-risk myelodysplastic syndromes

In order to extend existing outcome parameters in the older LR-MDS population, ${ }^{21}$ RBCT administration in the LR-MDS patients enrolled in the EUMDS Registry was 
evaluated with the aim of estimating the prognostic impact of regular RBCT and to validate RBCT-free survival as an early clinical endpoint in this patient population. The reference endpoint was progression-free survival (PFS), because both progression and death indicate the end of a relatively stable phase of LR-MDS.

A cohort of 1,267 patients with all relevant data available was included in this analysis. The patients were subdivided for the landmark analysis into four groups: no transfusions, $>0$ to $<0.75$ units/month (low transfusion dose), 0.75-1.75 units/month (mid transfusion dose) and $>1.75$ units/month (high transfusion dose). The greatest effect, compared to the non-transfused patients, occurred in patients receiving transfusions at low dose densities, since the impaired outcome of the mid and high transfusion density group was similar (Figure 2). In multivariable analysis, RBCT dose density retained statistical significance at $P<10^{-4}$.

Since treatment with ESA, lenalidomide and iron chelators may improve erythropoiesis and reduce the need for RBCT, these variables were included in the regression model. This analysis resulted in an effect for the dose density similar to the previous analyses at $P<10^{-4} .{ }^{21}$ However, the dose density effect continues to increase beyond one unit per month after correction for the three interventions (ESA, iron chelation and lenalidomide) up until a dose of six units per month (Figure 3). The relative log ratios on PFS of this analysis clearly showed that the deleterious effect of transfusions already occurred at a very low transfusion burden $(<3$ units per 16 weeks as defined in the revised International Working Group, IWG) report, confirming the outcome of the landmark analysis (see above). It is important to realize that patients with a transfusion dose of 1-2 units per 16 weeks are considered to be untransfused in the recently revised IWG report, but are recommended to be studied in future clinical trials. ${ }^{21}$

\section{Relevance of patient-reported outcomes in lower-risk myelodysplastic syndromes}

Health related quality of life is an important patientreported outcome (PRO). It provides specific information on
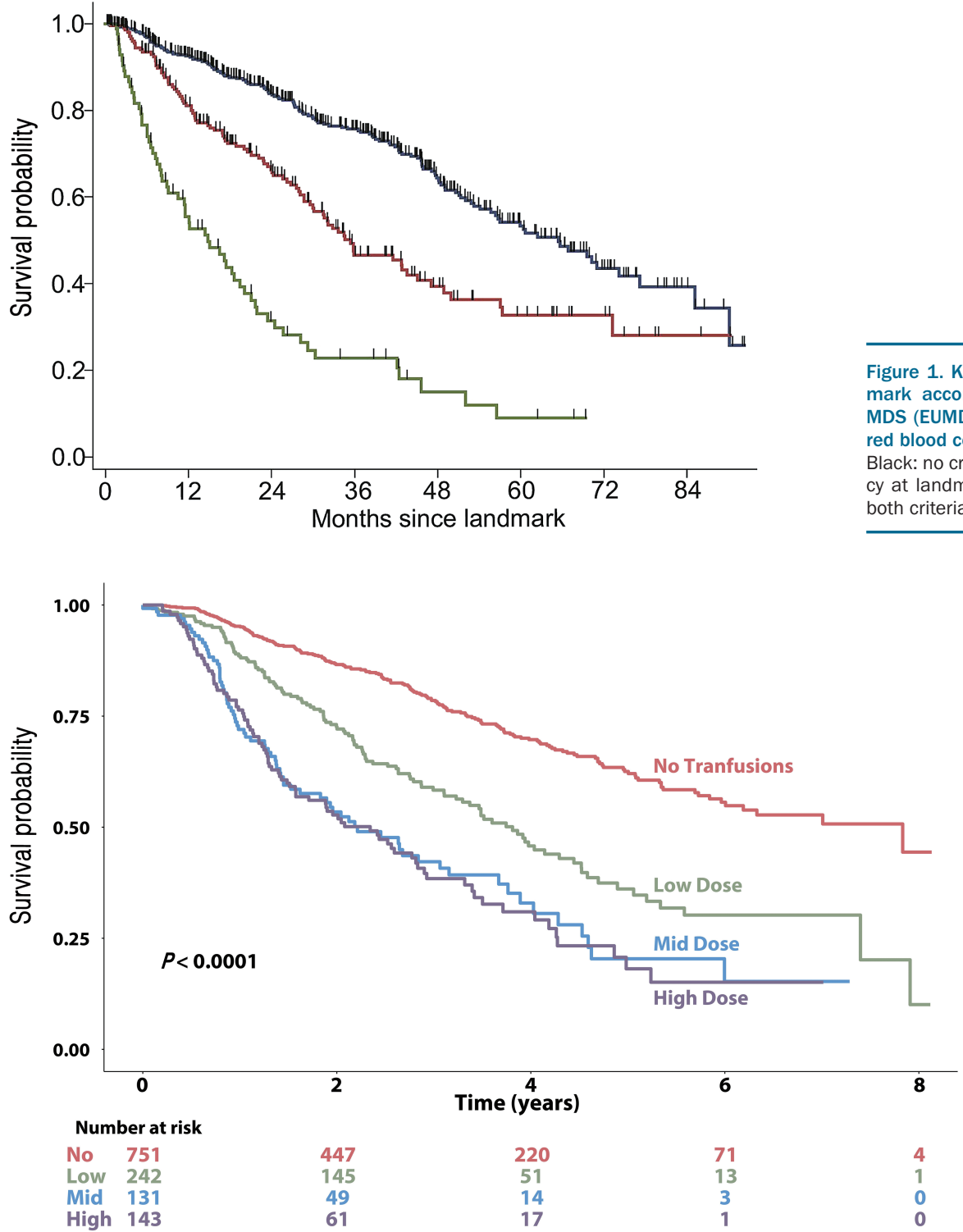

Figure 1. Kaplan-Meier plots of overall survival from landmark according to the 6-month European LeukemiaNet MDS (EUMDS) classifier based on platelet drop $>25 \%$ and red blood cell transfusion (RBCT)-dependency at landmark. Black: no criteria (no platelet drop $>25 \%$ or RBCT-dependency at landmark); red: either one of the two criteria; green: both criteria.

Figure 2. Kaplan-Meier plots of progressionfree survival (PFS) according to transfusion status at the landmark of visit 3 ( 1 year after registration). Kaplan -Meier plot of PFS of patients receiving no transfusions (red line) or transfusions at a low dose density: from $>0$ to $<0.75$ units per month (green line); mid dose density: $0.75-1.75$ units per month (purple line); high dose density $>1.75$ units per month (blue line). 
older patients with chronic diseases that might not be reflected in the level of physical activity and relevant laboratory parameters, including $\mathrm{Hb}$ levels. ${ }^{22}$ Preliminary data in HR-MDS patients suggest that these limitations may predict an unfavorable clinical outcome. ${ }^{8}$ However, definitive data on HRQoL in LR-MDS are rare. This EUMDS Registry project investigated the HRQoL-profile of LR-MDS patients at time of diagnosis as compared with age- and sex-matched reference groups from the general population. ${ }^{23} \mathrm{HRQoL}$ was measured by the EuroQol-5 dimension (EQ-5D) score at the time of study enrolment. ${ }^{24,25}$ Population norms were used to assess the relative HRQoL of patients in comparison to those of the average person in the community. ${ }^{26}$

A significant proportion of MDS patients reported moderate or severe problems in the dimensions pain/discomfort $(50 \%)$, mobility (41\%), anxiety/depression (38\%), and usual activities (36\%). Clinically meaningful restrictions in the EQ-5D index, EQ-Visual Analog Scale (VAS) were observed significantly more often in older patients and in those with a high co-morbidity burden, low $\mathrm{Hb}$ levels or RBCT need $\left(P<10^{-3}\right)$. Relative to the EQ-5D index and EQVAS scores in the reference group, HRQoL was significantly lower for groups of patients with MDS who were older, female, or had increased comorbidities, low Hb-levels or RBCT dependence. ${ }^{23}$

Restrictions in distinct dimensions of the EQ-5D were also observed when compared with European reference populations, but this effect might (partly) be explained by the anemia in the MDS cohort, since older anemic patients in the general population also have a decreased HRQoL. ${ }^{11}$ Low $\mathrm{Hb}$ levels and RBCT need were associated both with a decreased EQ-5D index and a decreased EQ-VAS after adjustment for co-variables in this EUMDS-Registry study, further supporting the use of RBCT requirement as an indicator of loss of effectiveness of interventions and worsening outcome. In addition, transfusion-free survival appeared to be a meaningful clinical endpoint in patients with lowerrisk MDS, as shown in the transfusion study. ${ }^{21}$ These findings have important implications for every-day clinical practice and the design of clinical endpoints.

\section{Novel treatment-response indicators in lower risk myelodysplastic syndromes}

Early initiation of treatment with erythropoietin stimulating agents is an important response indicator and significantly delays the onset of red blood cell transfusion dependency in patients with lower-risk myelodysplastic syndromes

Anemia of patients with LR-MDS and RBCT dependency have been associated with reduced HRQoL in several prospective trials $s^{27,28}$ and with reduced survival in retrospective registry reports. ${ }^{10}$ Current guidelines recommend ESA as first-line treatment for LR-MDS patients with symptomatic anemia. ${ }^{13}$ In recent studies, overall response rates varied between $38 \%$ and $66 \%$, with a median response duration of around 20 months. ${ }^{29}$ Retrospective analyses of large multi-center cohorts from different countries compared survival in patients treated with ESA within clinical studies with untreated patients. Survival was markedly better in the group exposed to ESA with no difference in AML transformation. . $^{30,31}$

Within the EUMDS registry, the effects of ESA treatment on outcomes were explored amongst 1,696 unselected patients with anemia. ${ }^{32}$ To overcome potential confounding by non-random allocation of ESA treatment, proportional hazards regression models comparing time-to-event outcomes in treated and untreated patients were weighted by stabilized inverse probability of treatment weights based on the propensity of a patient to receive ESA treatment. Only patients with comparable propensity scores were included in the analyses to estimate the effects of ESA treatment on outcomes. The relationship between the effects of ESA and pre-ESA treatment transfusion status was explored using this model. A non-significant beneficial effect of ESA treatment on OS was estimated from the weighted regression model comparing patients with comparable propensity scores (hazard ratio [HR] 0.82, 95\% confidence interval [CI]: 0.65-1.04; $P=0.09$ ). A non-significant estimate of a beneficial effect of ESA treatment on progression to AML or

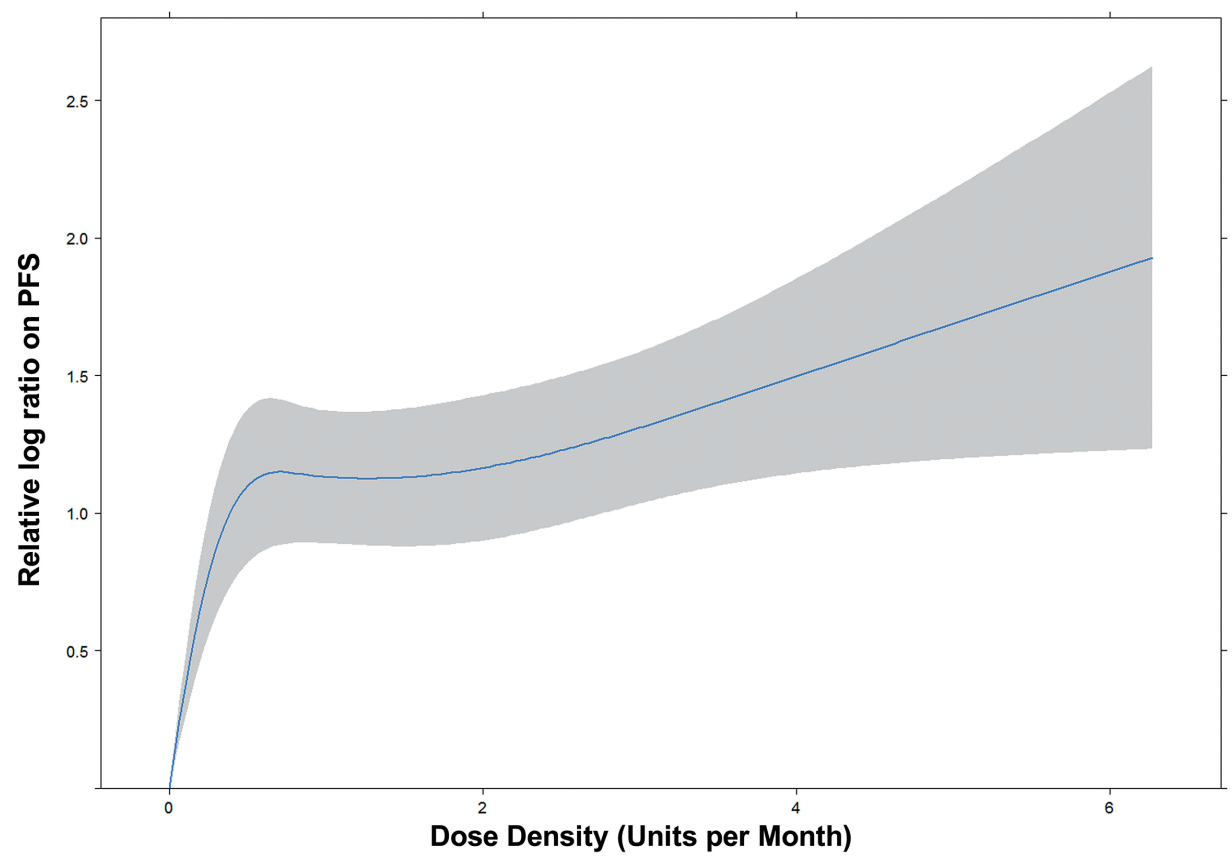

Figure 3. Influence of dose density on progression-free survival (PFS) in a multivariate regression model adjusted for treatment with either erythropoiesis-stimulating agent, iron chelation or lenalidomide. Dose density: average number of transfusions per month, calculated from start of transfusion until date of analysis. 
high-risk MDS was observed (HR 0.88, CI: 0.63-1.22; $P=0.44)$. Exploration of the relationship between ESA treatment and pre-treatment transfusion status revealed a larger estimated effect of ESA on survival amongst patients who had not received RBCT prior to starting ESA treatment (HR $0.71,95 \%$ CI: $0.49-1.03 ; P=0.07$ ) than amongst patients who had received prior RBCT (HR 0.93, 95\%CI: 0.70-1.26; $P=0.67)$.

Responding patients had a better prognosis, in terms of a lower risk for death (HR 0.65, 95\% CI: 0.45-0.893; $P=0.018$ ). The effect of response on time to first post-ESA treatment transfusion was significant after stratification by pre-treatment transfusion experience. Importantly, and irrespective of response status, patients who received RBCT before starting ESA had a shorter time to their first post-treatment transfusion (median 6.1 vs. 23.3 months for non-transfused patients; HR 2.4, 95\% CI: $1.75-3.31 ; P<10^{-4}$ ).

This large observational study showed that the response rate to ESA, as well as the capacity of these agents to significantly delay the onset of a regular RBCT need, is most pronounced in RBCT-naive patients, suggesting that RBCTnaive patients are more responsive. These results identify early initiation of ESA treatment as a relevant treatment response indicator, and suggest that ESA should be recommended as first-line treatment in LR-MDS patients with symptomatic anemia before starting regular RBCT.

\section{Labile plasma iron levels and non-transferrin bound iron are early and clinically relevant indicators of iron toxicity and impact of iron chelation therapy on outcome in patients with lower-risk myelodysplastic syndromes receiving red blood cell transfusion}

The majority of LR-MDS patients become RBCT dependent over time. With an expected survival of up to 12 years, these patients are prone to long-term accumulation of iron due to RBCT. ${ }^{33}$ Iron overload may also occur in a fraction of MDS patients who do not receive RBCT, resulting from the stimulation of intestinal iron absorption, mediated through suppression of hepcidin production by ineffective erythropoiesis. ${ }^{34}$ The toxic effects of iron overload in other iron loading diseases are well known, but the consequences in $\mathrm{MDS}$ remain to be elucidated. To this end, we evaluated erythroid marrow activity, hepcidin levels, and body iron status, including non-transferrin bound iron (NTBI) and labile plasma iron (LPI) levels over time in LRMDS patients and their relation with disease subtype and RBCT history within the EUMDS Registry. ${ }^{35}$

Detectable NTBI already occurred in all patient groups at registration, with highest levels in patients with MDS and ring sideroblasts (MDS-RS). The median LPI levels were below the level of detection in all patient groups at registration, except in transfusion dependent (TD) MDS-RS patients. ${ }^{35}$ Hepcidin levels increased with the number of transfused units, but in contrast, hepcidin levels significantly decreased over time in transfusion independent (TI) MDS-RS patients. Serum transferrin (sTfR) levels increased significantly over time in both TI and TD MDS-RS patients $\left(P\right.$-values from 0.01 to $\left.<10^{3}\right)$. Both elevated NTBI and LPI levels showed a threshold effect with transferrin saturation (TSAT) rates of $>70 \%$ and $>80 \%$, respectively. Elevated LPI levels occurred almost exclusively in patients with MDS-RS and/or patients, who had received RBCT. Once LPI levels are increased, survival time decreases, with greatest impact in patients who are TD (adjusted HR, 4.03, 95\%CI: 0.9517.06; $P=0.06$ ).
This study among LR-MDS patients showed that both treatment with RBCT and presence of ring sideroblasts increased the occurrence of the toxic iron species NTBI and LPI in serum. These data suggest that body iron accumulation and toxic iron species (NTBI and LPI) occur mainly in MDS-RS patients along the axis of ineffective erythropoiesis, characterized by elevated sTfR, low hepcidin, and increased iron levels, in some MDS subtypes, irrespective of receiving RBCT. Transfusional parenchymal iron overload, reflected by the combination of high serum ferritin levels, as well as direct iron toxicity, reflected by the presence of NTBI and LPI, was noted more frequently in MDS patients with ring sideroblasts compared to patients without ring sideroblasts. These data show that elevated LPI levels were associated with decreased survival both in the overall population of this study and in the patient groups subdivided by RBCT status. This implies that the widely used parameter TSAT cannot serve as a parameter to predict survival; however, TSAT rates can be used as a pre-screening marker to identify patients who are at risk of developing elevated LPI levels and associated poor prognosis. Finally, we could demonstrate in a limited number of patients treated with iron chelators that LPI levels decreased below detectable levels. This study suggested that NTBI and LPI may serve as early indicators of iron toxicity and as a measure for the effectiveness of iron chelation therapy in patients with lower risk MDS.

Iron overload due to RBCT is associated with increased morbidity and mortality in patients with LR-MDS. ${ }^{36}$ Several studies have reported beneficial effects of ICT on survival and other clinical outcomes in MDS patients with iron overload. ${ }^{37,38}$ However, valid data on the effect of ICT are limited since most studies are executed in small, selected patient groups or suffer from serious methodological problems such as confounding by indication. ${ }^{38}$ Performing a randomized, controlled trial (RCT) for this research question is awkward, and patients included in RCT may not reflect the general LR-MDS patients, who are usually patients of advanced age with multiple chronic, complex comorbidities. In addition to the possible beneficial effects of ICT on survival, increasing evidence indicates hematologic improvement in patients during ICT. ${ }^{39}$ Following improvement in cytopenias, transfusion independency is achieved in a minority of chelated patients. ${ }^{40,41}$

Results from a study conducted within the EUMDS registry on 490 non-chelated and 199 chelated patients using ICT as a time-dependent variable showed that the hazard ratio for OS was 0.50 (95\%CI: 0.34-0.74) after adjusting for relevant confounding factors. Restriction of the analysis to 150 patients who were initially treated with deferasirox resulted in the adjusted HR for OS of 0.38 (95\%CI: $0.24-$ 0.60 ), while patients who were initially treated with deferoxamine had inferior OS compared to deferasirox treated patients (adjusted HR: 2.46, 95\%CI: 1.12-5.41). The propensity-score analysis matching for all relevant variables, and a multivariate Cox proportional hazard model restricted to the deferasirox treated patients resulted in the adjusted HR for OS of 0.34 (95\%CI: 0.22-0.53). An erythroid response occurred in 77 chelated patients: 61 patients had a reduction in transfusion density, and 16 patients who did not have a reduction in transfusion density became transfusion independent during at least one visit interval.

The TELESTO trial ${ }^{42}$ is the only prospective, randomized, placebo-controlled study of ICT in MDS patients comparing deferasirox with a placebo-control group. This study 
evaluated the event-free survival (EFS) (a composite outcome, including non-fatal events related to cardiac and liver function, and transformation to AML or death) and safety of deferasirox versus placebo in low and intermediate-1-risk MDS patients. This trial demonstrated an EFS risk reduction of $36.4 \%$ in the deferasirox arm $(P=0.015)$, but the median $O S$ in the deferasirox-treated arm did not differ (HR 0.83, 95\%CI: 0.54-1.28; $P=0.200)$ when compared with placebo. The results of the TELESTO study are in line with the EUMDS study, but the patients included may not represent 'real life' older MDS patients with multiple comorbidities, as reflected by the mean age of 61 years of the patients included in TELESTO study compared to the mean age of 70 years of the chelated patients in the EUMDS Registry study. Furthermore, low accrual rates and the cross-over to ICT after cessation of the placebo affected the statistical power of the TELESTO study.

\section{Summary and concluding remarks}

Available evidence suggests that in most patients with LR-MDS the risk of death is not related to disease progression but is mainly attributable to non-leukemic death., ${ }^{2,17}$ In addition, a proportion of these patients have prolonged survival that precludes the design of clinical trials adopting $O S$ as a primary endpoint. These challenges have resulted in potentially biased assessment of the effectiveness and appropriate use of the available interventions in this patient population. The EUMDS Registry has identified novel meaningful outcome indicators and clinical endpoints, and reliable measures of response to $\mathrm{HCI}$ (Figure 4).

The results of our analysis indicate that RBCT density is strongly associated with a decreased OS, even at relatively low dose densities. In addition, we observed that an early decrease in platelet count is an independent adverse prognostic indicator in LR-MDS, and combining relative platelet drop and transfusion dependency allows early identification of patients at risk of rapid progression, and may guide early therapeutic interventions, including allogeneic hematopoietic stem cell transplantation or experimental interventions. Taken together, these results indicate that regular RBCT requirement, early platelet count kinetics, and restriction in HRQoL are early independent and meaningful outcome indicators, and reliable measures of effectiveness of therapeutic interventions, evaluated in this set of studies. These findings support the integration of RBCT requirement and HRQoL in the general core outcome sets and in response criteria in patients with LR-MDS, and have important implications for clinical practice and the design of clinical endpoints. Our results strongly support the adoption of freedom from transfusion as a meaningful clinical endpoint in patients with LR-MDS.

Anemia is the main determinant of therapeutic intervention in patients with LR-MDS, and ESA are recommended as first-line treatment for patients with symptomatic anemia. ${ }^{10}$ The observational studies within the EUMDS Registry showed that the response rate, as well as the capacity of these agents to delay the onset of a regular RBCT need, is most pronounced in RBCT-naive patients. These results identified early initiation of treatment with ESA as a major treatment response indicator, and indicate that ESA should be recommended in LR-MDS patients with symptomatic anemia before starting regular RBCT. After the onset of RBCT dependency, patients with LRMDS are prone to long-term accumulation of iron. ${ }^{1,43}$ The EUMDS Registry studies provided evidence that elevated LPI levels are associated with reduced survival in RBCT

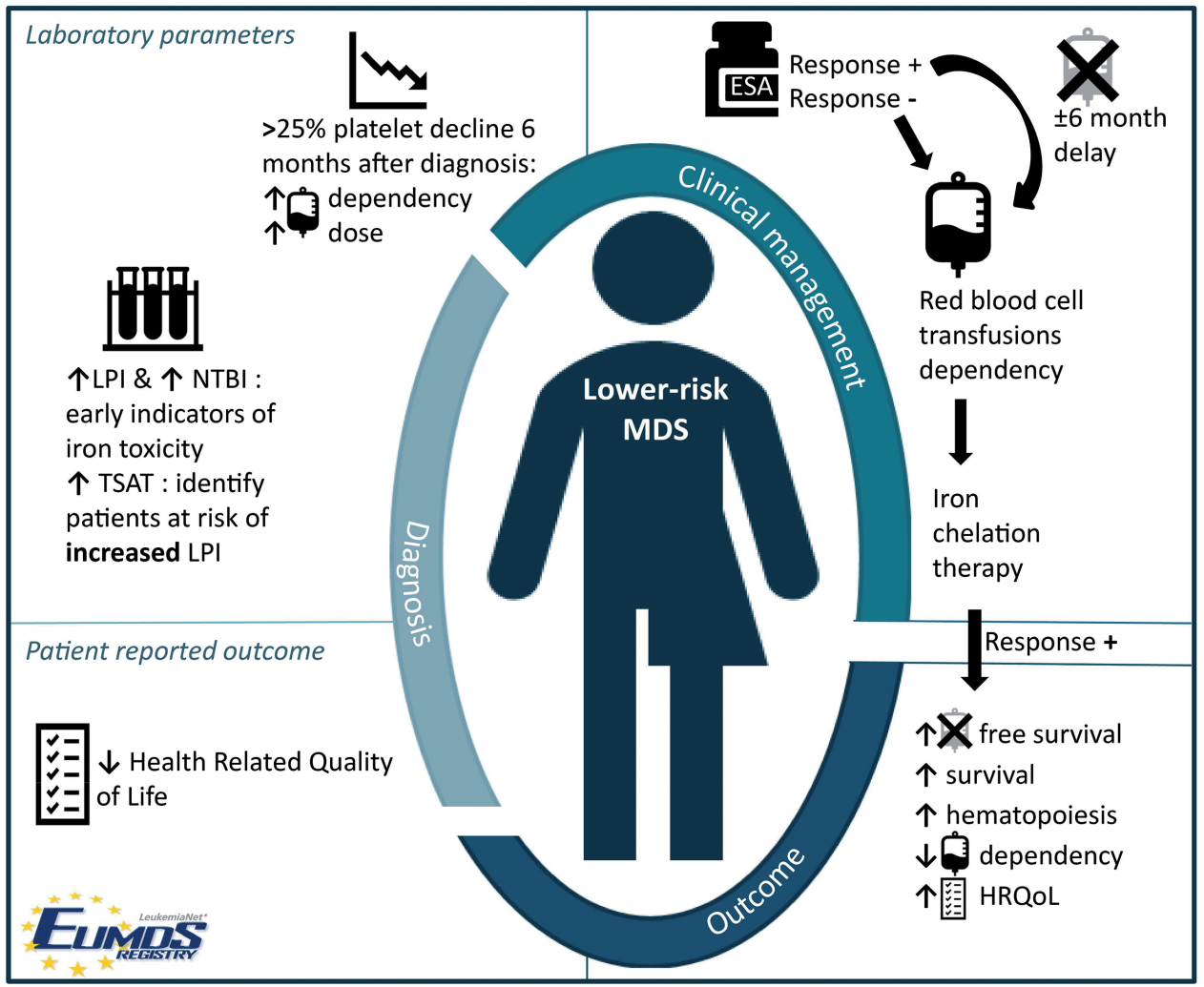

Figure 4. Overview of novel outcome indicators and clinical endpoints identified in the European LeukemiaNet myelodysplastic syndromes (EUMDS) Registry. ESA: erythropoietin stimulating agents; LPI: labile plasma iron; NTBI: non-transferrin bound iron; TSAT: transferrin saturation; HRQoL: health related quality of life. 
dependent patients, whereas iron chelation therapy normalizes LPI levels. These findings suggest that NTBI and LPI may serve as early indicators of iron toxicity and a means to measure the effectiveness of iron chelation therapy in patients with LR-MDS. However, qualified NTBI and LPI are only currently available in specialized laboratories. $^{44}$

Large observational cohorts with detailed clinical and laboratory data, like the EUMDS cohort, are the ideal framework in which to identify well defined MDS subtypes that may benefit from novel targeted treatments. An example of such a subtype is MDS with loss of parts of chromosome 5, namely del5q; these patients have a relatively favorable outcome on lenalidomide treatment. In order to identify homogeneous subsets of patients within MDS, preliminary evidence has suggested that recently identified mutations in splicing factors may recognize distinct disease entities within myeloid neoplasms. ${ }^{45}$ Splicing modulators are now in pre-clinical testing, and are very likely to lead to the introduction of effective drugs for specific groups of MDS patients. Luspatercept, a specific inhibitor of growth and differentiation factor-11, a member of the transforming growth factor $\beta$ superfamily, induced substantial improvement of anemia, especially in patients with ring sideroblasts. ${ }^{46}$ Characterization of individual cases by new genetic markers (one of the main objectives of the MDS-RIGHT project) will allow refined classification of patients into biological subgroups that are expected to respond differently to therapeutic interventions to guide discontinuation of those interventions that are less effective or less cost-effective.

The main question is whether RCT data and retrospective cohort data in selected tertiary care centers are representative of the 'real world' data of the older patients with LR$\mathrm{MDS}$ in the general population. A careful comparison of the 'real world' data and the RCT data will be needed in order to provide a clear answer to these questions. Meanwhile, the current analyses of data collected over 10 years in the EUMDS Registry provides relevant and important information which could help assess prognosis and response to standard interventions in this older patient group.

\section{Acknowledgments}

The authors would like to thank all local investigators (full list in Annex 1), operational team members, data managers, research nurses and patients for their contribution to the EUMDS Registry; Nicole Blijlevens, Jackie Droste and the research group at Radboud University Medical Center, Nijmegen for administrative, logistical and practical support; Dorine Swinkels, Rian Roelofs, and Erwin Wiegerinck of the Radboudumc expertise center for iron disorders for the measurement of LPI, NTBI and hepcidin-25; Jan Verhagen for his contribution in the measurement of the iron parameters; Margot Rekers, Karin van der Linden, and Siem Klaver for sample handling; Elise van Pinxten-van Orsouw and Linda van der Landen for data entry of all iron parameters; Erica Travaglino, and Chiara Elena for accruing patients and validation of the Pavia Registry data; and Tim Bagguley, W. Thomas Johnston, Louise de Swart for their contribution to the analyses. We would like to express specific thanks to Odile Beyne-Rauzy, Fabio Efficace, Otilia Georgescu, Njetoĉka Gredelj-Šimec, Agnes Guerci-Bresler, Gerwin Huls, Karin A. Koinig, Marian van Kraaij, Marta Krejci, Elisa Luño, Mac Macheta, Marius MacKenzie, Slobodanka Ostojić-Kolonić, Panagiotis Panagiotidis, Sophie Park, Chloé Reiniers, Borhane Slama, and Michail Spanoudakis for their contribution to the studies described, and Rosalie Lubber for the design of Figure 4.

\section{Funding}

The work of the EUMDS Registry is supported by an educational grant from Novartis Pharmacy B.V. Oncology Europe, Amgen Limited, and Celgene. This work is part of the MDSRIGHT activities, which has received funding from the European Union's Horizon 2020 research and innovation programme under grant agreement n. 634789 - "Providing the right care to the right patient with MyeloDysplastic Syndrome at the right time". The Pavia Registry is supported by a grant from Associazione Italiana per la Ricerca sul Cancro (IG 20125). Part of the work is supported by Translational Implementation of genetic evidence in the management of MDS (TRIAGE-MDS) (TRIAGE-MDS, Austrian Science Found I 1576) within the TRANSCAN - Primary and secondary prevention of cancer call (ERA Net).

\section{References}

1. Cazzola M, Malcovati L. Myelodysplastic syndromes-coping with ineffective hematopoiesis. $\mathrm{N}$ Engl J Med. 2005;352(6):536-538

2. de Swart L, Smith A, Johnston TW, et al. Validation of the revised international prognostic scoring system (IPSS-R) in patients with lower-risk myelodysplastic syndromes: a report from the prospective European LeukaemiaNet MDS (EUMDS) registry. Br J Haematol. 2015;170(3):372-383.

3. Greenberg P, Cox C, LeBeau MM, et al. International scoring system for evaluating prognosis in myelodysplastic syndromes. Blood. 1997;89(6):2079-2088.

4. Greenberg PL. Molecular and genetic features of myelodysplastic syndromes. Int J Lab Hematol. 2012;34(3):215-222.

5. Malcovati L, Porta MG, Pascutto C, et al. Prognostic factors and life expectancy in myelodysplastic syndromes classified according to WHO criteria: a basis for clinical decision making. J Clin Oncol. 2005;23(30):7594-7603.

6. Goldberg SL, Chen E, Corral M, et al. Incidence and clinical complications of myelodysplastic syndromes among United States Medicare beneficiaries. J Clin Oncol. 2010;28(17):2847-2852.

7. Della Porta MG, Malcovati L, Strupp C, et al. Risk stratification based on both disease status and extra-hematologic comorbidities in patients with myelodysplastic syndrome. Haematologica. 2011;96(3):441-449.

8. Efficace F, Gaidano G, Breccia M, et al. Prognostic value of self-reported fatigue on overall survival in patients with myelodysplastic syndromes: a multicentre, prospective, observational, cohort study. Lancet Oncol. 2015;16(15):1506-1514.

9. Efficace F, Gaidano G, Breccia M, et al. Prevalence, severity and correlates of fatigue in newly diagnosed patients with myelodysplastic syndromes. $\mathrm{Br} \mathrm{J}$ Haematol. 2015;168(3):361-370.
10. Malcovati L, Della Porta MG, Strupp C, et al Impact of the degree of anemia on the outcome of patients with myelodysplastic syndrome and its integration into the WHO classification-based Prognostic Scoring System (WPSS). Haematologica. 2011;96(10):1433-1440.

11. Wouters $H$, van der Klauw MM, de Witte $T$, et al. Association of anemia with healthrelated quality of life and survival: a large population-based cohort study. Haematologica. 2019;104(3):468-476.

12. Malcovati L, Galli A, Travaglino E, et al. Clinical significance of somatic mutation in unexplained blood cytopenia. Blood. 2017;129(25):3371-3378.

13. Malcovati L, Hellstrom-Lindberg E, Bowen $\mathrm{D}$, et al. Diagnosis and treatment of primary myelodysplastic syndromes in adults: recommendations from the European LeukemiaNet. Blood. 2013;122(17):29432964.

14. Hellstrom-Lindberg E, Gulbrandsen N, Lindberg G, et al. A validated decision 


$$
\mathrm{TI}
$$

